\title{
MICROCANTILEVERS FOR THERMAL NANOIMAGING AND THERMOMECHANICAL SURFACE MODIFICATION
}

\author{
William P. King, Thomas W. Kenny, and Kenneth E. Goodson \\ Department of Mechanical Engineering, Stanford University \\ Stanford, CA 94305-3030 \\ Michel Despont, Urs Duerig, Mark Lantz, Hugo Rothuizen, Gerd Binnig, and Peter Vettiger \\ IBM Research Division, Zurich Research Laboratory \\ Rueschlikon, Switzerland, CH-8803
}

\begin{abstract}
This paper reports recent advancements in the development of a microelectromechanical systems (MEMS) based data storage system that uses an array of heated atomic force microscope (AFM) cantilevers to write, read, and erase nanometer-scale data bits in thin polymer film. The cantilever tip contacts the polymer and induces highly local heating and melting to form data bit indentations with a radius of curvature near $20 \mathrm{~nm}$. The same cantilever can be used to thermally detect the presence of previously written bits through operation as an anemometer. This paper reports detailed measurement of cantilever heating for understanding and improving reading and writing operation, improved understanding of the bit formation process that makes possible data density near $0.9 \mathrm{Tbit} / \mathrm{in}^{2}$, the realization of a singlebit erasing process that employs the highly local melting in the polymer during data writing, and detailed measurement of the thermal reading process. The controlled writing, reading, and erasing of data bits reported in this paper complete the basic technological requirements for the development of a data storage system.
\end{abstract}

\section{INTRODUCTION}

The ultimate limits of magnetic data storage technology will be governed by thermal limits on magnetic data bit size $[\mathbf{1 , 2}]$ and mechanical positioning of the reading and writing heads [2]. It is at present unclear what technological path will overcome these limitations and permit data densities of $1 \mathrm{Tbit} / \mathrm{in}^{2}$ and beyond. Approaches to overcome media-induced limits on large data densities include perpendicular recording [3], recording on patterned media [3], and thermally-assisted recording [4]. MEMSbased data storage could help overcome mechanical alignment and packaging issues through systems integration [5]. Furthermore, MEMS-based data storage approaches that use scanning probes [6,7] could help overcome mechanical registration and tracking issues. This paper reports progress in the development of a MEMS data storage system based on scanning probes.

Thermomechanical data storage uses a heated, scanning atomic force microscope (AFM) cantilever tip to melt indentations into a polymer film [7-10]. Bit detection is possible by measuring temperature changes of the heated cantilever as it scans over a previously written surface [7-10]. Figure 1 shows a schematic of thermomechanical writing and thermal reading. Fig. 2 shows images of fabricated cantilevers. A complete data storage system will reliably and deliberately write, read, and erase data bits. A data density of $400 \mathrm{~Gb} / \mathrm{in}^{2}[\mathbf{8}]$ has been the maximum data density reported for thermomechanical data storage. The previously reported erasing scheme heated the entire chip to melt and reflow the polymer bit indentations and thus forcing a $100 \%$ erase of the media. Recent improvements in understanding heat transfer during reading and writing allow further increases in data density, $[\mathbf{1 1}, \mathbf{1 2}]$ improved sensitivity in data reading, $[\mathbf{1 0}]$ and design of a single-bit erase. We have recently reported design of cantilevers $[\mathbf{1 0}]$ and cantilever arrays [12] for data writing and reading. This paper reports further developments: increased data density by a factor of 2.5 to near 1 Tbit/in ${ }^{2}$, single-bit erasing, and quantitative data reading.

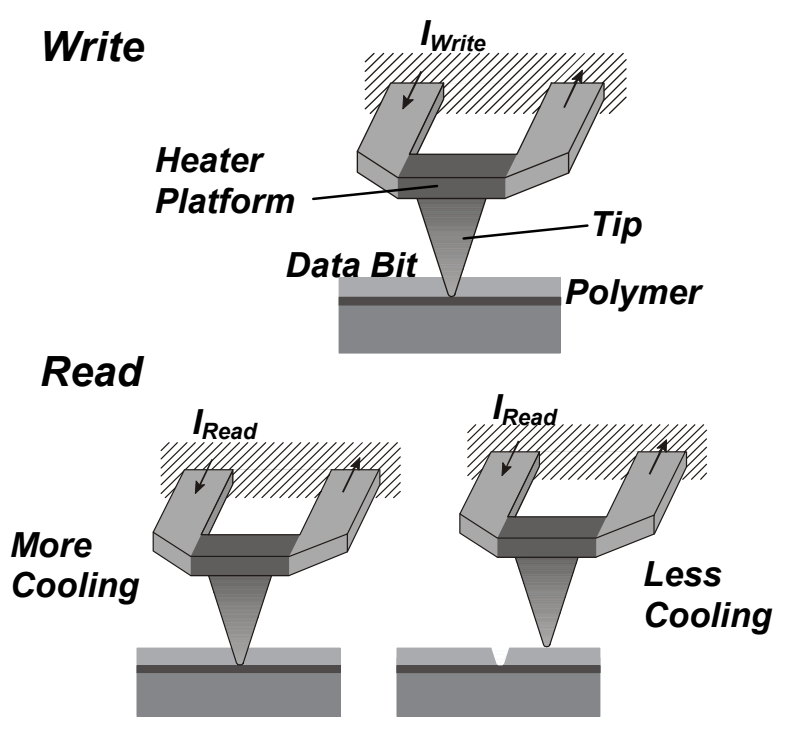

Figure 1. Thermomechanical data writing and reading.
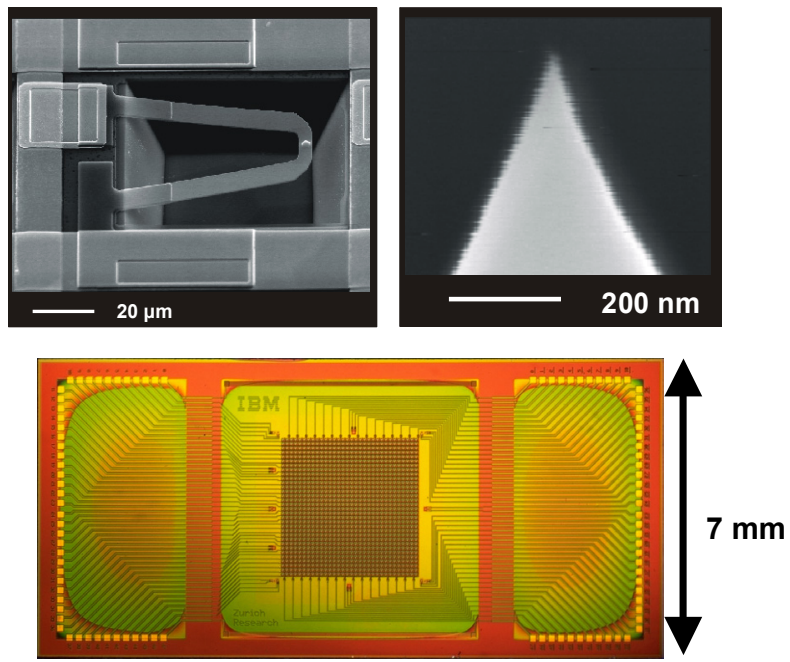

Figure 2. Scanning electron microscope images of the cantilever, cantilever tip, and a photograph of the cantilever array. Fabrication details are reported in [13]. 


\section{CANTILEVER OPERATION}

Previous measurement [9] and modeling [10-12] have shown that cantilever heater temperature and the power dissipated in the cantilever govern the size and shape of the data bits and the sensitivity of thermal reading. In order to monitor the power dissipated in the cantilever and the temperature in the cantilever heater platform, the cantilever with resistance $R_{C}$ is always operated in series with a resistor, $R_{S}$, shown in Fig. 3. Cantilever characterization and bit writing measurements are made with just the cantilever and a single series resistor. Also shown in Fig. 3 is a feedback control circuit used for thermal reading operation. The amplifier holds the bridge voltage $V_{0}=V_{\text {OFFET }}$ for a 'constant voltage' type operation. A voltage pulse of duration $t$ and amplitude $V_{+}-V_{0}$ is applied to the cantilever, where typical values are $1-100 \mu$ s and $0-3 \mathrm{~V}$. The output of the control circuit is the cantilever voltage integrated over some integration time $t_{i}$. The integration time is the time over which the cantilever electrical response is measured, and is not necessarily the cantilever heating time. The cantilever cools after each cantilever heating event, and the circuit resets for the next measurement.

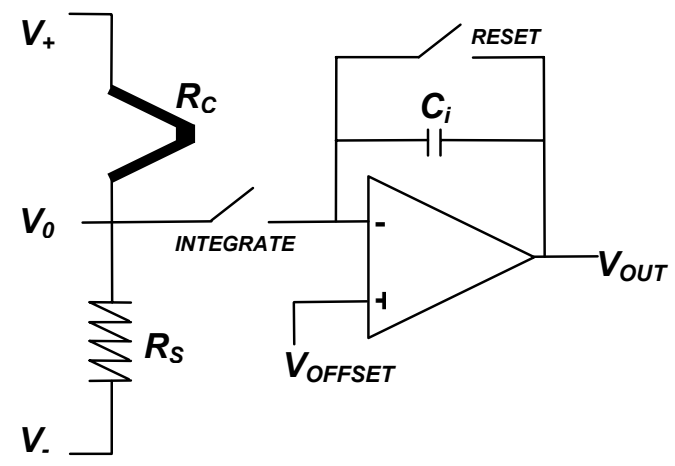

Figure 3. Schematic of cantilever driving circuit.

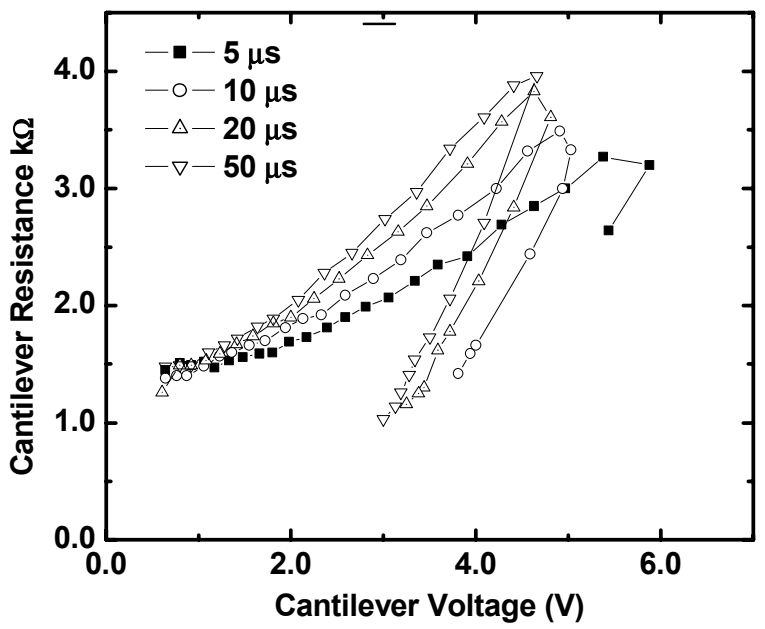

Figure 4. Cantilever electrical resistance at the end of short voltage pulses.

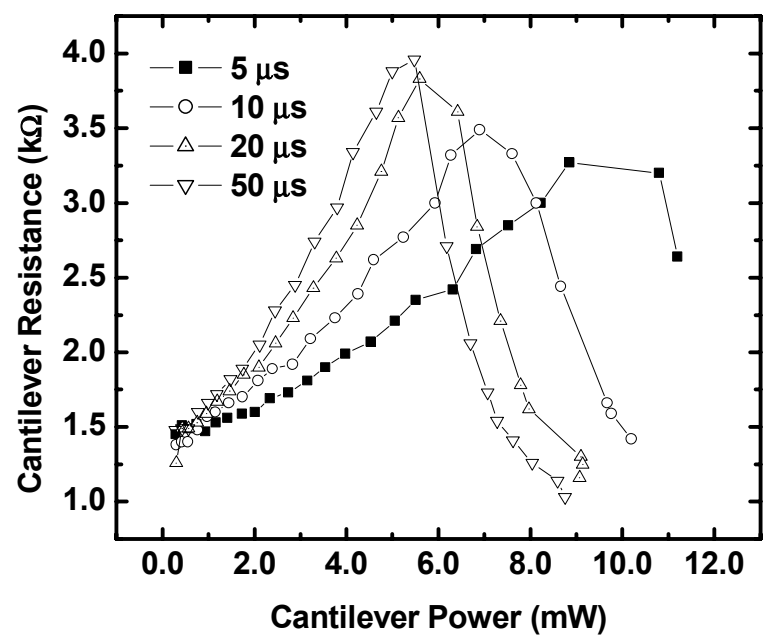

Figure 5. Power dissipated in cantilever at the end of short voltage pulses.

Figure 4 shows the cantilever electrical resistance at the end of short electrical pulses for a range of cantilever voltages and heating times and $R_{S}=3 \mathrm{~kW}$. The 'corner' effect is due to intrinsic carrier generation at high temperatures. Heat diffuses along the length of the cantilever in approximately $20 \mu \mathrm{s}$, which accounts for the large change in R-V characteristics at less than 20 $\mu \mathrm{s}$. Figure 5 shows the cantilever electrical resistance as a function of power dissipated in the cantilever. For long heating times, the cantilever power increases linearly with the cantilever heater temperature.

\section{DATA BIT WRITING AND ERASING}

Modeling [11,12] and measurement [9] of data bit writing has identified the spread of heat in the polymer layer as the fundamental limit on data bit density. Figure 6 shows a topographic map of thermomechanically written data bits. The sharp depressions below the polymer surface are the bit centers. The ring surrounding each is the polymer displaced during writing, more clearly shown in Fig. 7.

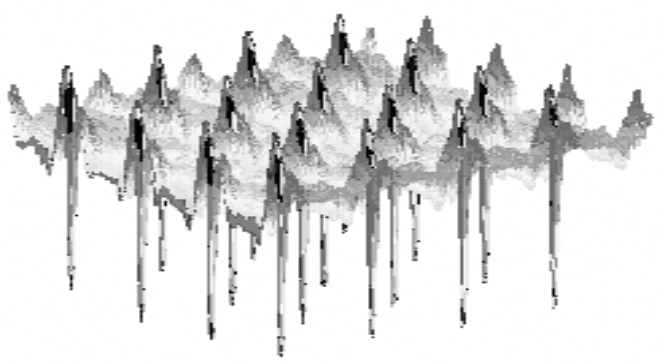

Figure 6. Field of thermomechanically written data bits. The deep centers of the bits and the conservation of mass pileup ring around the bits are clearly noticeable. The z-axis is unconditioned thermal reading signal and the field is $3 \mu \mathrm{m} X 3 \mu \mathrm{m}$ in size.. 


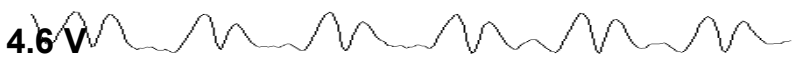

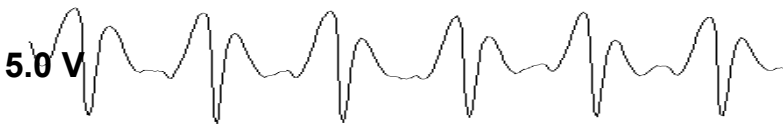

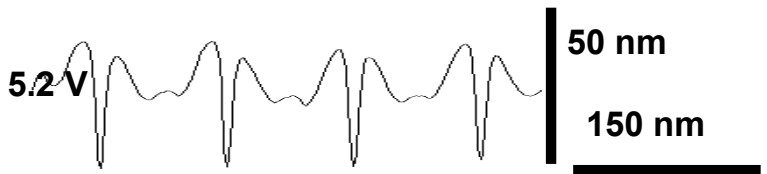

Figure 7. Line scan across six data bits written at four different writing conditions. The cantilever tip loading force was $30 \mathrm{nN}$, the writing time was $10 \mu \mathrm{s}$. Again, the bit centers and pileup rings are clear.

Modeling and simulation of heat transfer in the polymer layer during data bit formation shows that heat spreads away from the tip, softening a radial heat-affected zone near the tip [11,12]. Our hypothesis is that the dimensions of the pileup ring structure surrounding each bit are in fact the dimensions of melted polymer in proximity to the heated tip. Thus, bits written very close to each other or within the pileup ring of a previously written bit will influence the shape of neighbor bits.

To test this hypothesis, we perform an experiment where bits are written at increasing spatial periodicity. Figure 8 shows the results. As the bits are written successively closer together, they begin to melt and deform neighbor bits. For the highest spatial periodicity, the writing nearly eliminates previous bits. The ultimate data density of this technology will therefore depend upon the bit periodicity limited by this highly local melting. For the tip shape, polymer thickness, and writing conditions in Fig. 8, there is a maximum data density of $0.9 \mathrm{Tbit} / \mathrm{in}^{2}$.

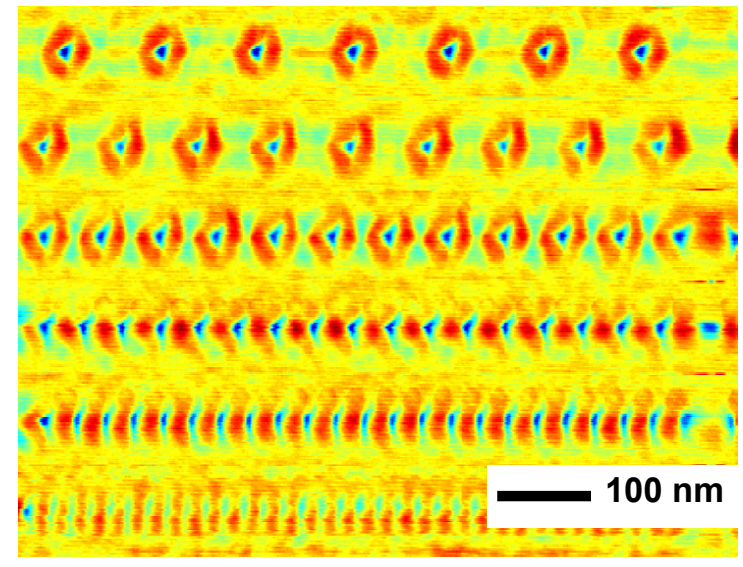

Figure 8. Data bits written with varying spatial periodicity. The melting of the polymer near each bit limits bit proximity. For these writing conditions, the limit on bit pitch is approximately $30 \mathrm{~nm}$, which corresponds to $0.9 \mathrm{Tbit} / \mathrm{in}^{2}$.

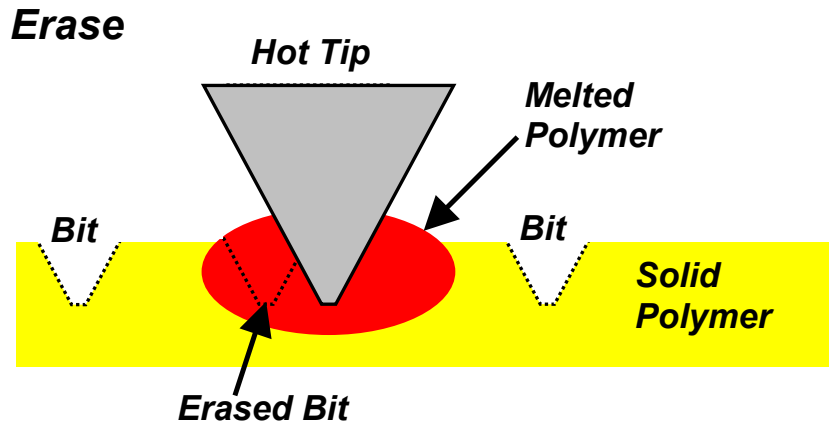

Figure 9. Concept schematic of data bit erasing. As the melted polymer region increases in size and spreads away from the tip, the melting acts to erase nearby bits.

While local melting limits data density, it offers an opportunity for local erasing. Previous erasing schemes [1,3] employed global erasing where the whole polymer substrate was heated to melt and reflow the entire polymer media substrate. The highly local melted near the heated tip during bit formation allows for local erasing, illustrated in Fig. 9.

Figure 10 shows images of bits written and then erased on the same location on the polymer data substrate. Erasing is accomplished by over-writing with a $15 \mathrm{~nm}$ bit pitch along the line of the written bits. As erasing requires over-writing, one bit will always be left at the end of a line of erased bits. This bit could be used to store information, depending upon its location, or be ignored, depending upon the data encoding scheme used.

Figure 11 shows line scans across the center line of the written/erased bits. We have demonstrated more than $10^{5}$ writeerase cycles with no measurable change in the required writing conditions or the data reading signal. However, it should be noted that mechanical wear of the tip [14] and relaxation of the polymer data bits at high temperatures and long times [15] will impact the operation of a data storage device over its lifetime.

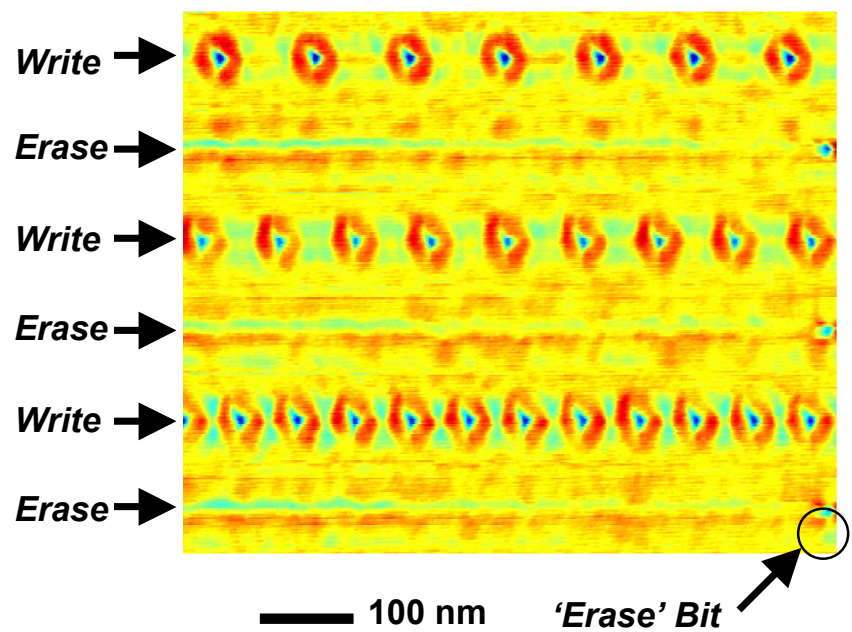

Figure 10. Three lines of successive writing and erasing on the same polymer 'real estate'. The erased regions were made by overwriting with a dense bit pitch of $15 \mathrm{~nm}$. As erasing requires multiple writes, the last erase write remains as a single 'erase' bit at the end. 


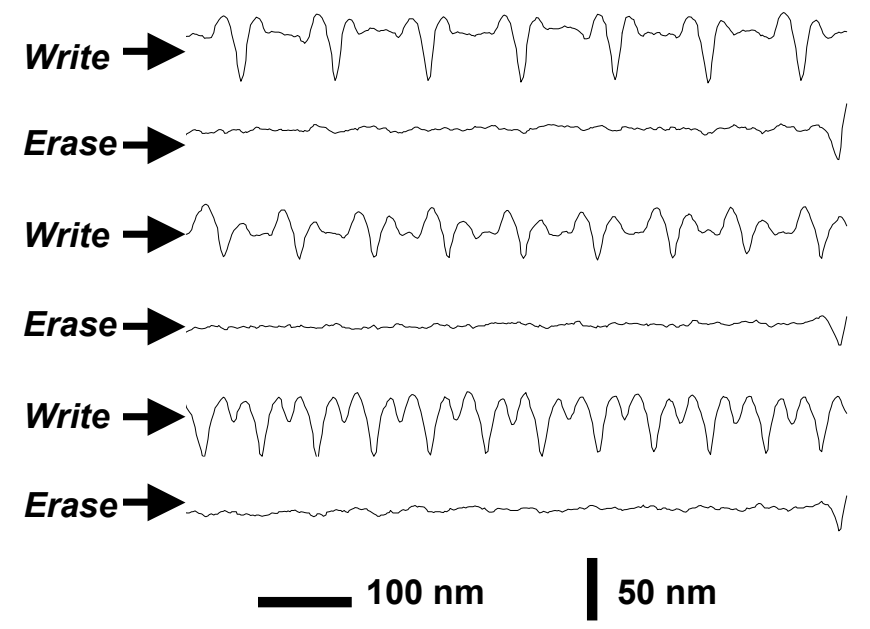

Figure 11. Line scans of the three lines of successive writing and erasing shown in Fig. 10.

\section{THERMAL READING}

Previous approaches to thermal data reading reported cantilever sensitivity in the range of $0.001 \mathrm{~nm}^{-1}$ for a near steadystate reading operation $[\mathbf{8 - 1 0}]$. While offering interesting opportunities for nanometer-scale sensing, a steady-state type operation where the cantilever is constantly heated is not practical for a data storage system. This paper reports a thermal data reading technique that measures the cantilever response to short electrical pulses, which is a function of the thermal impedance between the cantilever and the substrate. The control circuit of Fig. 3 integrates the cantilever reading signal over the heating pulse, thus increasing the cantilever signal over that of the steady-state reading technique. This pulsed-heating reading technique also allows for integration with digital circuitry, which is necessary for a data storage system.

The cantilever reading sensitivity is the dependence of the measured cantilever electrical signal on feature height of the polymer data substrate. The cantilever electrical and thermal properties, as well as the integration. The $\Delta R / R$ cantilever sensitivity is measured for reading of data bits of depth $50 \mathrm{~nm}$. The difference between measured maximum and minimum signals yields the quantity $\Delta R$, and the measured signal at the neutral polymer surface gives the denominator $R$. In pulsed-heating operation, the cantilever signal integration yields a gain of

$$
G=\frac{t_{i}}{\left(R_{C}{ }^{-1}+R_{S}^{-1}\right)^{-1} C_{i}}
$$

Where $G$ is gain, and $C_{i}$ is the capacitance of the integration capacitor, shown in Fig 3. For typical integration times of $5-50$ $\mu$ s, values for $R_{L}$ and $R_{S}$ of $\sim 3 \mathrm{k} \Omega$, and $C \sim 100 \mathrm{pF}$, typical values for $G$ are in the range 50-500. All of the measurements shown here for pulsed-heating reading are normalized with respect to $G$ so that they may be compared with the steady-state reading, for which $G=1$.

All of the images and data in this paper are made at an integration reset time of $3 \mathrm{kHz}$, which is sufficiently long for the cantilever to cool, and for the cantilever scanner to move over the polymer. For all of the measurements here, integration time $t_{i}$ is equal to half the heating time, made over the second half of the heating pulse.

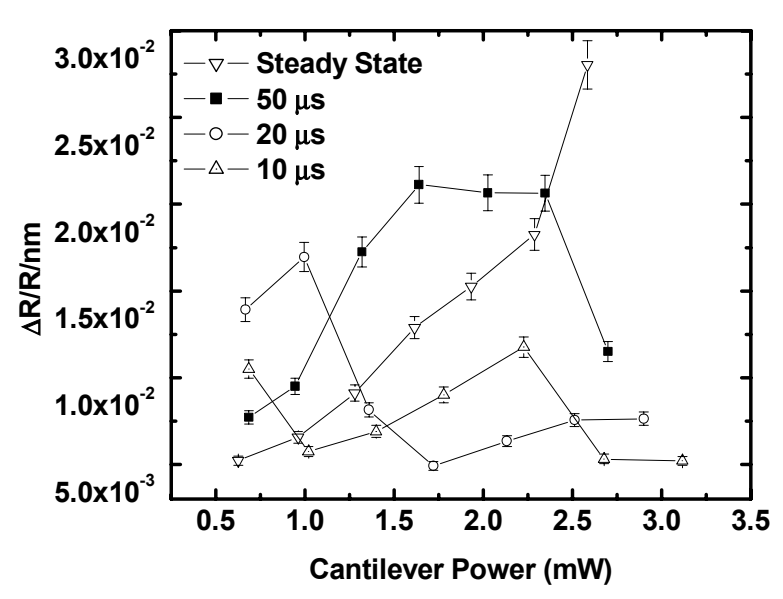

Figure 12. Cantilever reading sensitivity as a function of heating power and reading time.

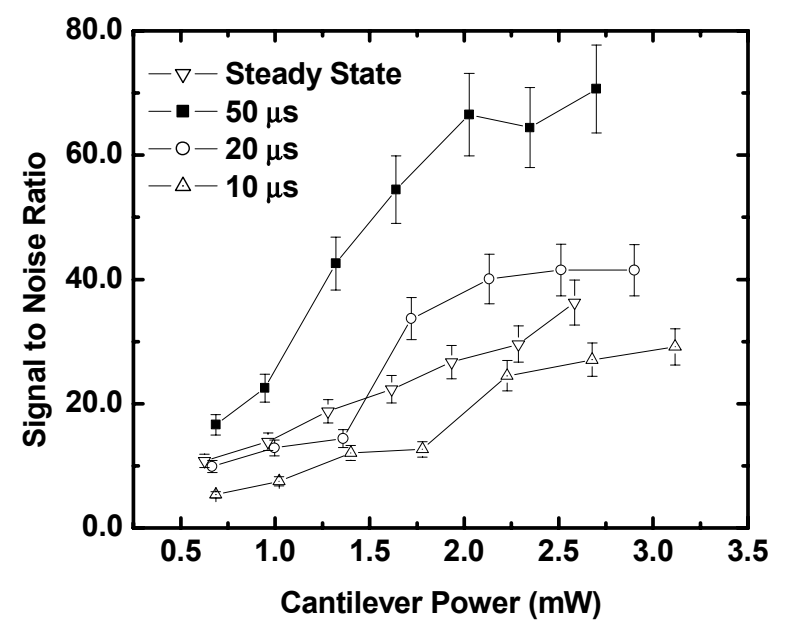

Figure 13. Signal-to-noise ratio (SNR) of thermal reading of 50 $n m$ data bits as a function of heating power and reading time.

Calibration of the thermal impedance measurement allows quantitative measurement of the vertical features of Figs. 8 and 10. Figure 12 shows the cantilever sensitivity as a function of heating power and time. The cantilever $\Delta R / R$ vertical sensitivity is as high as $0.003 \mathrm{~nm}^{-1}$ in steady state operation, requiring $2.5 \mathrm{~mW}$ of power. The cantilever is more sensitive for longer times as the cantilever relaxes to thermal equilibrium. While increased power increases sensitivity in steady state operation, it does not always correspond to increased sensitivity in pulsed operation. Close comparison of Figs. 4 and 5 with Fig. 12 helps explain this result. The slopes of the $R-V$ and the $R-P$ curves are much steeper for longer heating times than shorter heating times. Furthermore, for heating times of less than $\sim 20 \mu \mathrm{s}$, the cantilever legs do not heat substantially and therefore do not assist in the thermal reading process.

Figure 13 shows the cantilever signal-to-noise (SNR) characteristics for measuring $50 \mathrm{~nm}$ data bits, with SNR as high as 70. The signal is calculated as for Fig 12, and the noise is the root-mean-squared noise measured as the cantilever scans over the unmodified polymer substrate. The dominant noise sources are the cantilever and the media, with instrumentation noise measured as comparatively small. Pulses of $\sim 20 \mu$ s or longer are required to 
achieve SNR better than steady-state operation, as this is the time required for heat to diffuse the length of the cantilever.

\section{CONCLUSIONS}

This paper uses improved understanding of the heat transfer processes during thermomechanical data bit writing to achieve data density of $0.9 \mathrm{Tbit} / \mathrm{in} 2$, demonstrate local data bit erasing, and measure figures of merit for the thermal data reading process.

Along with previously reported design [5,6], fabrication [3], and operation $[1,6]$ of cantilevers and cantilever arrays, the controlled writing, reading, and erasing of data bits reported in this paper complete the basic technological requirements for the development of a data storage system.

Future work will explore the optimization of tip shape, heating time, heating temperature, loading force, and polymer thickness. There are also interesting opportunities in using the cantilever for nanoscale thermal processing, manufacturing, and materials characterization at the nanometer scale. The very high thermal data reading sensitivity suggests that there may be applications for sub-nanometer displacement sensing.

\section{ACKNOWLEGEMENTS}

WPK appreciates the support of IBM Zurich Research Laboratory and the IBM Graduate Research Fellowship Program. The authors further thank B. Gottsmann and P. Seidler of IBM Zurich Research, T. Albrecht of IBM Almaden Research, and B. Chui of Stanford University.

\section{REFERENCES}

1. E. Grochowski and R. F. Hoyt, "Future Trends in Hard Disk Drives," IEEE Transactions Magnetics, 32, 1850 (1996).

2. D. A. Thompson and J. D. best, "The Future of Magnetic Data Storage Technology," IBM Journal of Research and Development, 44, 311 (2000).

3. J. Lohau, A. Moser, C. T. Rettner, M. E. Best, and B. D. Terris, "Writing and reading perpendicular magnetic recording media patterned by a focused ion beam," Applied Physics Letters, 78, 990 (2001).

4. J. J. M. Ruigrok, R. Coehoorn, S. R. Cumpson, and H. W. Kesteren, "Disk recording beyond 100 Gb/in.': Hybrid Recording?," Journal of Applied Physics, 87, 5389 (2000).

5. L. R. Carley, J. A. Bain, G. K. Fedder, D. W. Greve, D. F. Guillou, M. S. C. Lu, T. Mukherjee, S. Santhanam, L. Abelman, and S. Min, "Single-Chip Computers with Microelectromechanical Systems-Based Magnetic Memory," Journal of Applied Physics, 87, 6680 (2000).

6. H. J. Mamin, R. P. Ried, B. D. Terris, and D. Rugar, "HighDensity Data Storage Based on the Atomic Force Microscope," Proceedings of the IEEE, 87, 1014 (1999).

7. P. Vettiger, M. Despont, U. Drechsler, U. Dürig, W. Häberle, M. Lutwyche, H. E. Rothuizen, R. Stutz, R. Widmer, and G. Binnig, "The "Millipede" - More than one thousand tips for future AFM data storage," IBM Journal of Research and Development, 44, 323 (2000).
8. G. Binnig, M. Despont, U. Drechsler, W. Häberle, M. Lutwyche, P. Vettiger, H. J. Mamin, B. W. Chui, and T. W. Kenny, "Ultrahigh-Density Atomic Force Microscopy Data Storage with Erase Capability," Applied Physics Letters, 76, 1329 (1999).

9. G.L.W. Cross, M. Despont, U. Drechsler, U. Dürig, P. Vettiger, W.P. King, and K.E. Goodson, "Thermomechanical Formation and Thermal Sensing of Nanometer-Scale Indentations in PMMA Thin Films for Parallel and Dense AFM Data Storage," in Fundamentals of Nanoindentation and Nanotribology II, Materials Research Society Symposium Proceedings Vol. 649, 2001.

10. W. P. King, T. W. Kenny, K. E. Goodson, G. L. W. Cross, M. Despont, U. Durig, H. Rothuizen, G. Binnig, and P. Vettiger,

"Atomic Force Microscope Cantilevers for Combined Thermomechanical Data Writing and Reading," Applied Physics Letters, 78, 1300 (2001).

11. W. P. King and K. E. Goodson, "Modeling and Simulation of Nanometer-Scale Thermomechanical Data Bit Formation," Proceedings of the ASME National Heat Transfer Conference, Anaheim, CA, (2001).

\section{W.P. King, PhD Thesis, Stanford University (2002).}

13. M. Despont, J. Brugger, U. Drechsler, U. Dürig, W. Häberle, M. Lutwyche, H. Rothuizen, R. Stutz, R. Widmer, H. Rohrer, G. K. Binnig, and P. Vettiger, "VLSI-NEMS Chip for Parallel AFM Data Storage," Sensors and Actuators A, 80, 100 (2000).

14. B. D. Terris, S. A. Rishton, H. J. Mamin, R. P. Ried, and D. Rugar, "Atomic Force Microscope-Based Data Storage: Track Servo and Wear Study," Applied Physics A, 66, S809 (1998).

15. U. Dürig, G. Cross, M. Despont, U. Drechsler, W. Haberle, M. I. Lutwyche, H. Rothuizen, R. Stutz, R. Widmer, P. Vettiger, G. K. Binnig, W. P. King, and K. E. Goodson, ""Millipede" : an AFM data storage system at the frontier of nanotribology," Tribology Letters, 9, 25 (2002). 